\title{
The prevalence and significance of anti-PM/Scl antibodies in systemic sclerosis
}

\author{
Ewa Wielosz ${ }^{1, A-F} \oplus$, Magdalena Dryglewska ${ }^{1, B} \oplus$, Maria Majdan ${ }^{1, A, F \oplus}$ \\ ${ }^{1}$ Department of Rheumatology and Connective Tissue Diseases, Medical University, Lublin, Poland \\ A - Research concept and design, B - Collection and/or assembly of data, C - Data analysis and interpretation, \\ $D$ - Writing the article, E - Critical revision of the article, F - Final approval of article \\ Wielosz E, Dryglewska M, Majdan M. The prevalence and significance of anti-PM/Scl antibodies in systemic sclerosis. Ann Agric Environ Med. \\ 2021; 28(1): 189-192. doi: 10.26444/aaem/127801
}

\begin{abstract}
Introduction. Anti-PM/Scl (a-PM/Scl) antibodies are found in different systemic autoimmune diseases such as polymyositis, dermatomyositis, systemic sclerosis (SSc) and overlap syndromes. According to literature, they are detected in approx. $2 \%$ of SSc patients, but their presence is more common in SSc with myositis overlap.

Objective. The aim of the study was to assess the prevalence of a-PM/Scl in patients with SSc and to identify differences in the clinical profile of the disease in patients with the presence of a-PM/Scl.

Materials and method. The study was performed on 126 European Caucasian SSc patients (98 females and 28 males) hospitalized consecutively in the Department of Rheumatology and Connective Tissue Diseases. The study group was analyzed for the potential presence of a-PM/Scl using a commercial test - EUROLINE Systemic Sclerosis Profile. The detection and interpretation were carried out electronically using the specific Euroimmun - EUROLineScan programme. The subtype of SSc, incidence of internal organ involvement and serological profile were determined in the entire group. Due to the presence of a-PM/Scl, patients were divided into two groups: a-PM/Scl (+) SSC - 22 patients and a-PM/Scl (-) SSC - 104 patients.

Results. A-PM/Scl was detected in 22/126 patients with SSc (17.5\%). A strong correlation was found between a-PM/Scl and myalgia or myositis $(p=0.0379)$, hand joints contractures $(p=0.0002)$ and the prevalence of overlap syndrome $(p=0.0142)$. There were no relationships between the presence of a-PM/Scl and subtypes of SSc, other organ involvement, digital ulcers or calcinosis.
\end{abstract}

Conclusions. Anti-PM/Scl antibodies are fairly common in patients with systemic sclerosis. In SSc, anti-PM/Scl antibodies are frequently associated with myalgia or myositis, hand joint contractures and an overlap syndrome.

\section{Key words}

systemic sclerosis, autoantibodies, anti-PM/Scl antibodies, serological profile

\section{INTRODUCTION}

In systemic sclerosis (SSc) autoantibodies against different extractable nuclear antigens can be present in patient sera. Clinical presentation and prognosis of SSc are known to be related to a particular serological profile. The main marker antibodies for SSc are anti-RNA polymerase III antibodies (a-RNA Pol III), anti-topoisomerase I antibodies (a-topo I) and anti-centromere antibodies (ACAs). They are associated with a typical subset and clinical manifestations of the disease $[1,2,3]$. Furthermore, they are listed among the ACR/EULAR classification criteria for systemic sclerosis [4]. ACAs are more common in limited cutaneous SSc (lcSSc) and are typically indicative of late-onset visceral involvement and better prognosis. A-topo I antibodies are reported in diffuse cutaneous SSc (dcSSc) and are worse prognostic factors of early onset of organ involvement. A-RNA Pol III antibodies signal progressive skin thickening, scleroderma renal crisis (SRC) and malignancy [5]. Other antibodies, such as anti-fibrillarin (a-U3RNP), anti-Th/To, and autoantibodies against nucleolus-organizing region-90 (a-NOR90), are also SSc specific antibodies, although their presence is less frequent. Moreover, the list of SSc-associated autoantibodies

Address for correspondence: Ewa Wielosz, Department of Rheumatology and Connective Tissue Diseases, Medical University, Jaczewskiego 8, 20-090, Lublin, Poland

E-mail: ewa.wielosz@wp.pl

Received: 13.05.2020; accepted: 10.09.2020; first published: 28.09.2020 includes anti-PDGFR, anti-PM/Scl (a-PM/Scl), anti-Ro52 and anti-Ku antibodies. Although these autoantibodies are characterized by lower frequency, they are also associated with the clinical course of systemic sclerosis [6, 7]. Apart from $\mathrm{SSc}$, a-PM/Scl antibodies are reported to occur in various systemic autoimmune diseases, including dermatomyositis (DM), polymyositis (PM), and overlap syndromes. They are directed against two major antigenic components: 100 and $75 \mathrm{kDa}$ proteins (a-PM/Scl 100 and a-PM/Scl 75). A-PM/Scl antibodies produce a nucleolar staining pattern [1].

\section{OBJECTIVE}

The aim of the study is to determine the incidence of a-PM/ $\mathrm{Scl}$ antibodies in SSc patients and to indicate dissimilarities in their individual clinical profiles.

\section{MATERIALS AND METHOD}

The study group consisted of 126 European Caucasian SSc patients (98 females and 28 males) undergoing treatment in the Department of Rheumatology and Connective Tissue Diseases. Overlap features of systemic sclerosis and other collagenosis were observed in 27 of the patients. As required by the Declaration of Helsinki, participants' informed consent was obtained and the study was approved by the Research 
Ethics Committee. The patients fulfilled the ACR and EULAR criteria for systemic sclerosis [4]. The subtype of SSc, organ involvement, mortality, and the serological profile were established. The patients were categorized as lcSSc or dcSSc according to Le Roy et al. [8]: 59 patients had dcSSc and 67 patients had lcSSc. Organ involvement was determined from clinical symptoms and diagnostic tests. Interstitial lung disease (ILD) was established by the presence of ground-glass opacification or bibasilar pulmonary fibrosis displayed in HRCT (High-Resolution Computed Tomography) scans. Lung function was assessed by DLCO (a percentage of predicted transfer factor for carbon monoxide) and TLC (total lung capacity) tests. Pulmonary hypertension (PAH) was indicated by the pulmonary arterial systolic pressure (sPAP) $>$ $35 \mathrm{mmHg}$, determined by Doppler echocardiography at rest. Cardiac involvement was inferred from the manifestations of conduction disturbances, arrhythmia or heart failure. The evidence for myositis or myalgia was muscle pain, muscle weakness and/or elevated serum creatine phosphokinase (CPK) levels. Accompanying articular involvement was diagnosed in the case of tender and swollen joints. Gastrointestinal system involvement was evidenced by subjective symptoms reported by the patients, such as heartburn, dysphagia, bloating or determined in a barium swallow test. Renal damage was designated by the presence of scleroderma renal crisis (SRC), elevated serum creatinine levels or proteinuria. Manifestations of digital ulcers and calcinosis were also assessed. Serum samples were obtained from each patient. Additionally, the presence of antibodies was determined with a commercial line immunoassay - EUROLINE Systemic Sclerosis Profile kit for detection of antibodies against scleroderma-specific antigens: a-RNA pol III, a-topo- I (anti-Scl-70), or ACAs, and less-frequent antibodies: anti-Ku, anti-Th/To anti-Ro52, a-PM/Scl subunits PM/Scl 100 and PM/Scl 75, anti-PDGFR and anti-NOR90. The serum samples were diluted 1:101 and subjected to antibody detection procedure with alkaline phosphatase-labeled antihuman IgG. The data were collected and analyzed electronically using the EUROLineScan system (EUROIMMUN. AG, Lübeck, Germany). Moreover, anti-double-stranded deoxyribonucleic acid antibodies (anti-dsDNA) were determined using enzyme-linked immunosorbent assay (ELISA) in the entire study group. The cohort was divided into two groups according to the incidence of a-PM/Scl antibodies: a-PM/Scl positive (+) SSc group - 22 patients and a-PM/Scl negative (-) SSc group - 104 patients.

The software package used for statistical analysis was Statistica 10.0, and the Chi-square test was employed to determine differences between variables in the group. The results were significant at the $P=0.05$ level.

\section{RESULTS}

A-PM/Scl antibodies were isolated in 22/126 SSc subjects (17.5\%), of whom 13 had a-PM/Scl-100 antibodies and 14 a-PM/Scl-75, including five patients positive for both. Clinical characteristics of a-PM/Scl positive (+) and a-PM/Scl negative (-) SSc patients are given in Table 1 . With respect to the serological parameters in the a-PM/Scl positive (+) SSc group, isolated a-PM/Scl antibodies were detected in 10 subjects, while in 12 the coexistence of SSc marker antibodies, such as ACA, a-topo-I and a-RNA pol III was detected. Moreover, in two cases, less-frequent antibodies were additionally found (a-
$\mathrm{Ku}$ - in 1 and a-U3RNP - in one patient). Table 2 compares the serological parameters in a-PM/Scl positive (+) SSc and a-PM/ $\mathrm{Scl}$ negative (-) SSc groups. Analyzing clinical manifestations of the disease in the study group, it was revealed that the incidences of myalgia, myositis and hand joints contractures were significantly more frequent in the a-PM/Scl positive (+) SSc group compared to the a-PM/Scl negative (-) SSc group (respectively $31.8 \%$ vs. $13.5 \%$; $P=0.038$ and $36.4 \%$ vs $7.7 \%$; $P=0.0002)$. It was shown that $9 / 22$ patients from the a-PM/Scl positive (+) SSc group had overlap syndrome, among them 7/9 - with polymyositis, and 2/9 with dermatomyositis overlapping systemic sclerosis. In the group of overlap syndromes, 4/9 had two different units of protein antigen. Furthermore, 2/9 patients tested negative for SSc marker antibodies.

To sum up, the results of the correlational analysis revealed a strong relationship between a-PM/Scl antibodies and the presence of overlap syndromes $(P=0.014)$; however, no associations were found when correlated according to antibodies directed against two different units of protein antigen. No correlations were shown between the occurrence of a-PM/Scl antibodies and the SSc subtypes, organ damage, digital ulcers, calcinosis or malignancy (Tab. 3).

Table 1. Clinical characteristics of patients in $\mathrm{SSC}$ a-PM/Scl positive (+) and SSC a-PM/Scl negative (-) groups

\begin{tabular}{lccc}
\hline & SSc a-PM/Scl (+) & SSc a-PM/Scl (-) & $p$ \\
\hline No. of patients & 22 & 104 & \\
dcSSc & $7(31.8 \%)$ & $52(50 \%)$ & \\
IcSSc & $15(68.2 \%)$ & $52(50 \%)$ & \\
\hline Gender & & & \\
$\quad$ Female & 17 & 81 & \\
$\quad$ Male & 5 & 23 & \\
\hline Age (years) & $48.8 \pm 16.9$ & $54.82 \pm 12.52$ & NS \\
\hline Duration of disease (years) & $(20.0-76.0)$ & $(19-81)$ & \\
\hline
\end{tabular}

Time from onset of Raynaud phenomenon to diagnosis (years)

$6.0 \pm 6.6(0.5-23.0) \quad 5.5 \pm 6.6(0.0-30.0) \quad$ NS

dcSSc - diffuse cutaneous systemic sclerosis; IcSSc - limited cutaneous systemic sclerosis. Data are presented as number and percentage. $P$-value of $<0.05$ was considered statistically significant.

Table 2. Comparison of selected serological parameters in $\mathrm{PM} / \mathrm{Scl}$ positive $(+)$ and PM/Scl negative (-) group of patients with systemic sclerosis

\begin{tabular}{lccc}
\hline & $\begin{array}{c}\text { SSc PmScl }(+) \\
\mathrm{n}=22\end{array}$ & $\begin{array}{c}\mathrm{SSc} \text { PmScl }(-) \\
\mathrm{n}=104\end{array}$ & $\mathrm{p}$ \\
\hline Anti-topo I (Scl-70) & $5(22.7 \%)$ & $43(41.3 \%)$ & $\mathrm{NS}$ \\
\hline Anti-centromere & $3(13.6 \%)$ & $23(22.1)$ & $\mathrm{NS}$ \\
\cline { 2 - 4 } CENPB & $3(13.6 \%)$ & $23(22.1 \%)$ & $\mathrm{NS}$ \\
CENPA & $2(9.1 \%)$ & $23(22.1 \%)$ & $\mathrm{NS}$ \\
\hline anti-RNA Polymerase & $4(18.2 \%)$ & $16(15.4)$ & $\mathrm{NS}$ \\
III RNA Pol III 11 & $3(14.3 \%)$ & $13(12.5 \%)$ & $\mathrm{NS}$ \\
RNA Pol III 155 & $2(9.5 \%)$ & $11(11.6 \%)$ & $\mathrm{NS}$ \\
\hline Anti-fibrillarin & $1(4.8 \%)$ & $2(1.9 \%)$ & $\mathrm{NS}$ \\
\hline Anti-Ku & $1(4.8 \%)$ & $3(2.9 \%)$ & $\mathrm{NS}$ \\
\hline Anti-Th/To & 0 & $2(1.9 \%)$ & $\mathrm{NS}$ \\
\hline Anti- Nor-90 & $1(4.8 \%)$ & $5(4.8 \%)$ & $\mathrm{NS}$ \\
\hline Anti-PDGFR & 0 & 0 & $\mathrm{NS}$ \\
\hline anti-Ro-52 & $4(4.8 \%)$ & $30(28.8 \%)$ & $\mathrm{NS}$ \\
\hline Anti-dsDNA & 0 & $3(2.9 \%)$ & $\mathrm{NS}$
\end{tabular}

Data are presented as number and percentage. $P$-value of $<0.05$ was considered statistically significant. 
Table 3. Comparison of selected clinical parameters in $\mathrm{PM} / \mathrm{Scl}$ positive $(+)$ and $\mathrm{PM} / \mathrm{Scl}$ negative (-) patients with systemic sclerosis

\begin{tabular}{|c|c|c|c|}
\hline & $\begin{array}{c}\text { SSc a-PmScl (+) } \\
n=22\end{array}$ & $\begin{array}{c}\text { SSc a-PmScl (-) } \\
n=104\end{array}$ & $\mathbf{p}$ \\
\hline ILD (HRCT) & $13(59 \%)$ & $53(51 \%)$ & NS \\
\hline Decreased DLCO & $14(61 \%)$ & $39(50 \%)$ & NS \\
\hline Heart involvement & $6(27.3 \%)$ & $40(38.5 \%)$ & NS \\
\hline PAH (ECHO) & $5(22.7 \%)$ & $22(21.1 \%)$ & NS \\
\hline Myalgia or myositis & $7(31.8 \%)$ & $14(13.5 \%)$ & 0.0379 \\
\hline Arthralgia & $18(81.8 \%)$ & $86(82.7 \%)$ & NS \\
\hline Arthritis & $10(45.5 \%)$ & $30(28.8 \%)$ & NS \\
\hline Gastrointestinal tract involvement & $13(59.1 \%)$ & $61(58.6 \%)$ & NS \\
\hline Renal involvement & $6(27.3 \%)$ & 30 (28.8\%) & NS \\
\hline SRC & $3(13.6 \%)$ & $6(5.8 \%)$ & NS \\
\hline Calcinosis & $6(27.3 \%)$ & $17(16.3 \%)$ & NS \\
\hline Digital ulcerations & $4(18.2 \%)$ & $20(19.2 \%)$ & NS \\
\hline Death & $2(9.1 \%)$ & $15(14.4 \%)$ & NS \\
\hline Overlap syndrome & $9(40.9 \%)$ & $18(17.3 \%)$ & 0.0142 \\
\hline Hand joints contractures & $8(36.4 \%)$ & $8(7.7 \%)$ & 0.0002 \\
\hline Malignancy & $2(9.1 \%)$ & $11(11 \%)$ & NS \\
\hline
\end{tabular}

ILD - interstitial lung disease; HRCT - high-resolution computer tomography; DLCO - diffusing capacity for carbon monoxide; PAH - pulmonary arterial hypertension; SRC - scleroderm renal crisis. Data are presented as number and percentage. $P$-value of $<0.05$ was considered statistically significant

\section{DISCUSSION}

Systemic sclerosis patients exhibit a high prevalence of a-PM/ $\mathrm{Scl}$ antibodies, which are detected in approximately $2-12 \%$ of SSc patients, while 7-8\% with PM and 1-2\% with DM. At the prevalence level of approximately $18 \%$, the results from this study suggest higher rates of a-PM/Scl antibodies in SSc patients than in other studies reported in the literature. This may result from detection methods, as it was suggested by the study of Jaskowski et al. [9]. Their results showed that despite the discrepancies between the correlation results from different a-PM/Scl antibody detection methodologies, the best agreement was displayed by immunoprecipitation and line immunoblot assays. Numerous studies have confirmed that further assay optimization and standardization efforts are advisable $[5,7,9]$. Additionally, the a-PM/Scl antibody rates are known to vary according to ethnic background, and are higher in Europeans and North Americans but lower in Asian patients $[1,10]$.

The observed discrepancy could also be associated with a high prevalence of overlap syndromes (17\%) in the current study group. The main overlapping diseases in this study were PM or DM (40\%) and a-PM/Scl antibodies strongly correlated with these overlaps. In fact, literature sources show that a-PM/Scl antibodies are currently widely associated with a high incidence of myositis, joint involvement and overlap syndrome in patients with SSc [11], and a-PM/Scl antibodies levels are higher in SSc with myositis overlap, reaching $25 \%$ [12]. Accordingly, the results revealed that myalgia or myositis and the presence of hand joint contractures were significantly more common in the positive a-PM/Scl (+) SSc group in comparison with the negative group. Furthermore, in the currentr study the mean age of a-PM/Scl positive (+) $\mathrm{SSc}$ patients was younger than that in the a-PM/Scl negative (-) SSc group; nonetheless, the difference was not found to be statistically significant. The literature data confirm these findings $[11,13,14]$.

Some authors compared the prevalence and clinical features in the a-PM/Scl-75 and a-PM/Scl-100 positive groups [11]. According to one large study, ina e group of 1,574 SSc patients, a-PM/Scl-75 antibodies were detected in 3\%, a-PM/Scl-100 in $1.1 \%$, and both in $1.7 \%$ of subjects [11]. However, only $1 \%$ had isolated a-PM/Scl-75 antibodies and $0.7 \%$ isolated a-PM/ Scl-100 antibodies, i.e. in isolation of each other and without concomitant presence of SSc-specific antibodies. These cases were associated with higher rates of calcinosis, whereas a higher prevalence of myositis was exclusively seen in patients positive for both a-PM/Scl-75 and a-PM/Scl-100 antibodies. In the same report, lung involvement was only observed in subjects with a-PM/Scl-75 and, in comparison, those with a-PM/Scl-100 antibodies showed higher survival rates [11].

The results from the current study show no major differences in the clinical profile of the disease when analyzed according to a-PM/Scl subunit. An explanation could be the small sample of patients. Likewise, in the overlap syndrome group, no correlation was found between the subunit of a-PM/Scl antibodies and PM or DM, although in this study group, 2/9 SSc patients with overlapping PM or DM had both a-PM/Scl-75 and PM/Scl-100 antibodies. Additionally, other clinical features are indicated as potentially positively correlated with a-PM/Scl antibodies, e.g. early onset of SSc or calcinosis $[15,16]$. Subcutaneous calcinosis is known to occur in a maximum of $58 \%$ of a-PM/Scl-positive vs. $30 \%$ of anti-PM/Scl-negative patients [12]. Moreover, a lower incidence of ILD and gastrointestinal symptoms were found in SSc patients with a-PM/Scl [15]. The presence of a-PM/ Scl antibodies in SSc patients with ILD has been reported to be associated with better lung parameters and reduced prevalence of restrictive pattern [15]. What is more, a-PM/Scl determines a milder course of $\mathrm{lcSS}_{\mathrm{c}}$ without substantial organ damage [12]. According to observations by the authors of the current study, there was no correlation between calcinosis, lcSSc subtype or lung involvement and presence of a-PM/ Scl antibodies. According to the authors' data, over $50 \%$ of patients in the a-PM/Scl positive (+) SSc group had other marker antibodies for SSc, such as ACAs, a-topo- I, and a-RNA pol III antibodies. In contrast, D'Aoust et al. reported that almost half of the 7\% of SSc patients showing a-PM/ Scl positivity, had no other SSc-specific antibodies [13]. In a different study, the coexistence of anti-dsDNA with a-PM/Scl antibodies was demonstrated; however, this combination of antibodies was rather infrequent (about $0.7 \%$ of connective tissue disease patients). Clinically, the a-dsDNA/a-PM/Scl group included a small number of SLE patients with cooccurrence of at least one SSc feature, including Raynaud's phenomenon and/or sclerodactyly [14]. In the presented SSc study group, the coexistence of a-dsDNA antibodies with a-PM/Scl antibodies was not confirmed.

There are also some controversial data reporting the association between a-PM/Scl antibodies and malignancy. A recent Spanish retrospective study involving 432 patients, including 53 cancers (12.2\%), found no association between a-RNA pol III antibodies and cancer and, in contrast, the a-PM/Scl antibody was associated with cancer with an OR of 3.90 (95\% CI 1.31-11.61) [17]. Moreover, Bruni C et al. showed that over $18 \%$ of a-PM/Scl positive SSc patients developed cancer [18]. Furthermore, according to Japanese data, DM subjects with a-PM/Scl antibodies exhibited a tendency to 
develop cancer $[19,20]$. These results, however, were not corroborated by a Dutch study [21]. The current analysis also did not indicate an association between malignancy and the presence of a-PM/Scl antibodies among SSc patients.

\section{CONCLUSIONS}

The results indicate that a-PM/Scl antibodies are commonly present in scleroderma patients. In approximately $40 \%$ of a-PM/Scl positive $(+)$ SSc cases, these antibodies could constitute an isolated serological marker of SSc, but in over $50 \%$ of patients, other SSc marker autoantibodies were found: ACAs, a-topo- I, or a-RNA pol III. A-PM/Scl antibodies in SSc typically indicate myositis, hand joint contractures and overlap syndrome. To sum up, measurements of a-PM/Scl antibodies prove useful in routine clinical practice as in many cases they identify the clinical profile and might predict the course of systemic sclerosis.

\section{REFERENCES}

1. Boonstra M, Mertens BJA, Bakker JA, et al. To what extent do autoantibodies help to identify high-risk patients in systemic sclerosis? Clin Exp Rheumatol. 2018; 36: 109-117.

2. Hamaguchi Y. Autoantibody profiles in systemic sclerosis: predictive value for clinical evaluation and prognosis. J Dermatol. 2010; 37: 42-53.

3. Shanmugam VK, Frech TM, Steen VD, et al. Collaborative National Quality and Efficacy Registry (CONQUER) for Scleroderma: outcomes from a multicenter US-based systemic sclerosis registry. Clin Rheumatol. 2020; 39: 93-102.

4. van den Hoogen F, Khanna D, Fransen J, et al. 2013 classification criteria for systemic sclerosis: an American college of rheumatology/European league against rheumatism collaborative initiative. Ann Rheum Dis. 2013; 72: 1747-1755.

5. Liaskos C, Marou E, Simopoulou T, et al. Disease-related autoantibody profile in patients with systemic sclerosis. Autoimmunity. 2017; 50: $414-421$.
6. Margot A, Smet J, Soyfoo S. Non-identified antinuclear antibodies in systemic sclerosis. Rev Med Brux. 2016; 37: 401-407.

7. Wielosz E, Dryglewska M, Majdan M. Serological profile of patients with systemic sclerosis. Postepy Hig Med Dosw. 2014; 18; 68: 987-991.

8. LeRoy EC, Black C, Fleishmajer R. Scleroderma (systemic sclerosis): classification, subsets and pathogenesis. J Rheumatol. 1988; 15: 202-204.

9. Jaskowski TD, Wilson A, Hill HR, Tebo AE. Diagnostic assays for antiPM/Scl IgG antibodies: heterogeneity in antibody response or lack of standardization? Clin Chim Acta. 2011; 12: 1100-1105.

10. Kohara A, Yanaba K, Muro Y, et al. Anti-PM/Scl antibody-positive dermatomyositis in a Japanese patient: a case report and review of the literature. Int J Rheum Dis. 2017; 20: 2186-2189.

11. Wodkowski M, Hudson M, Proudman S, et al. Clinical correlates of monospecific anti-PM75 and anti-PM100 antibodies in a tri-nation cohort of 1574 systemic sclerosis subjects. Autoimmunity. 2015; 48: 542-551.

12. Stochmal A, Czuwara J, Trojanowska M, Rudnicka L. Antinuclear Antibodies in Systemic Sclerosis: an Update. Clin Rev Allergy Immunol. 2020; 58: 40-51.

13. D'Aoust J, Hudson M, Tatibouet S, Wick J, et al. Clinical and serologic correlates of anti-PM/Scl antibodies in systemic sclerosis: a multicenter study of 763 patients. Arthritis Rheumatol. 2014; 66: 1608-1615.

14. Mahler M, Greidinger EL, Szmyrka M, et al. Serological and clinical characterization of anti-dsDNA and anti-PM/Scl double-positive patients. Ann N Y Acad Sci. 2007; 1109: 311-321.

15. Guillen-Del Castillo A, Pilar Simeón-Aznar C, et al. Good outcome of interstitial lung disease in patients with scleroderma associated to anti-PM/Scl antibody. Semin Arthritis Rheum. 2014; 44: 331-337.

16. Valenzuela A, Song P, Chung L. Calcinosis in scleroderma. Curr Opin Rheumatol. 2018; 30: 554-561.

17. Bernal-Bello D, de Tena JG, Guillén-Del Castillo A, et al. Novel risk factors related to cancer in scleroderma. Autoimmun Rev. 2017; 16: 461-468.

18. Bruni C, Lages A, Patel H, et al. Association of a-PM/Scl antibody with risk of malignancy in scleroderma. Rheumatol. 2015; 54: 160.

19. Muro Y, Hosono Y, Sugiura K, et al. Anti-PM/Scl antibodies are found in Japanese patients with various systemic autoimmune conditions besides myositis and scleroderma. Arthritis Res Ther. 2015; 11: 17:57.

20. Sada PR1, García LP, Rodrigo ME. Clinical heterogeneity of anti-PM/ Scl positive conditions. Br J Hosp Med (Lond). 2018; 79: 532-533.

21. Boonstra M, Huizinga TWJ. De Vries-Bouwstra JK. Auto-antibodies and cancer in systemic sclerosis. Autoimmun Rev. 2017; 16: 883-884. 